\title{
Visualization of Spatial Distribution of $\gamma$-Aminobutyric Acid in Eggplant (Solanum melongena) by Matrix-assisted Laser Desorption/Ionization Imaging Mass Spectrometry
}

\author{
Naoko Goto-Inoue, Mitsutoshi Setou, and Nobuhiro ZaIMA ${ }^{\dagger}$ \\ Department of Molecular Anatomy, Hamamatsu University School of Medicine, 1-20-1 Handayama, \\ Higashi, Hamamatsu, Shizuoka 431-3192, Japan
}

\begin{abstract}
We applied imaging mass spectrometry (IMS) to determine the spatial distribution of $\gamma$-aminobutyric acid (GABA). We found that GABA had a specific localization in seeds. We also visualized various biomolecules as well as GABA with higher spatial resolution than in the previous report. Our work suggests that IMS might be a powerful tool for exploring functional food factors, investigating the specific distribution of nutrients in unused natural resources, and evaluating the quality of functional foods.
\end{abstract}

(Received March 31, 2010; Accepted May 10, 2010; Published July 10, 2010)

\section{Introduction}

Molecular imaging techniques are essential for obtaining biological information. However, methods for visualizing endogenous small molecules remain to be established.

Approximately 200000 metabolites are thought to exist in plants. These metabolites play critical roles in biological functions, such as energy production and the biosynthesis of macromolecules. ${ }^{1}$ Numerous methods have been developed for their analysis in highly complex mixtures, namely mass spectrometry (MS) $)^{2-4}$ and MS coupled with separation techniques such as gas chromatography (GC), ${ }^{5}$ liquid chromatography (LC), ${ }^{6,7}$ and capillary electrophoresis (CE). ${ }^{3,8}$ Due to its inherently high sensitivity and molecular specificity, MS has become an important tool for the analysis of biological systems. In combination with the soft ionization method known as matrix-assisted laser desorption/ionization (MALDI), MS has proved to be useful in the investigation of biomolecules. A new imaging method using MALDI-MS, called imaging mass spectrometry (IMS), has been developed and is now widely used to visualize small molecules. IMS makes it possible to visualize the distribution of individual molecules in a tissue section without the use of antibodies, staining, or complicated pretreatment. ${ }^{9} 10$ Direct analysis of a tissue section using IMS allows the detection of a wide range of endogenous molecules such as lipids, ${ }^{10-14}$ glycolipids, ${ }^{15-17}$ and peptides, ${ }^{18,19}$ as well as the detection of administered pharmaceuticals. ${ }^{20,21}$ IMS might be adapted to the comprehensive analyses of various metabolites in plants.

There are many functional food factors and nutraceuticals around the world, and the number is increasing. Gamma-aminobutyric acid (GABA) is one of the most important functional food factors. Plants contain large amounts of GABA,

† To whom correspondence should be addressed.

E-mail: zaima@hama-med.ac.jp but the levels are highly variable in response to biotic and abiotic stresses, including acidic environments and mechanical damage, although the biological roles of GABA are still obscure. $^{22}$ GABA is involved in regulating plant development, protecting against oxidative stress, defending against insects, signaling, and regulating cytosolic $\mathrm{pH}$. In addition, GABA intake can help in the treatment of various neurological disorders. Knowing the specific distribution of GABA in plants is important for various fields of study, including nutrition and pharmacology, as well as in efforts to improve the production of GABA-rich plant cultivars. Previous studies utilized infrared (IR)-MALDI imaging ${ }^{23,24}$ and LDI-MALDI imaging ${ }^{25}$ to visualize small molecule distributions in plant sections. Various peaks, e.g., those of amino acids and sugars, were detected in the direct IR-MALDI imaging of plants. However, the machine used in that study was handmade and its spatial resolution was quite low. ${ }^{24}$ In this study, we applied IMS to visualize the GABA in eggplant sections by using conventional MALDI equipment with higher spatial resolution than is found with IR-MALDI imaging. Eggplant is botanically classified as a berry, and contains numerous small, soft seeds, which are edible, but also bitter because they contain a trace amount of nicotinoid alkaloids. Eggplant is known to contain little in the way of minerals or nutrition. It was cultivated from ancient times and is widely used as a popular vegetable around the world by virtue of its usefulness. Especially, eggplant contains large amounts of GABA. Thus we thought that eggplant would be an appropriate vegetable for visualizing GABA in plant tissue. Here we show the spatial distribution of GABA and other nutrition factors in eggplant.

\section{Experimental}

Reagents

Trifluoroacetic acid (TFA) and methanol were purchased from Kanto Chemical Company (Tokyo, Japan), and 2,5-dihydroxy- 
(a)
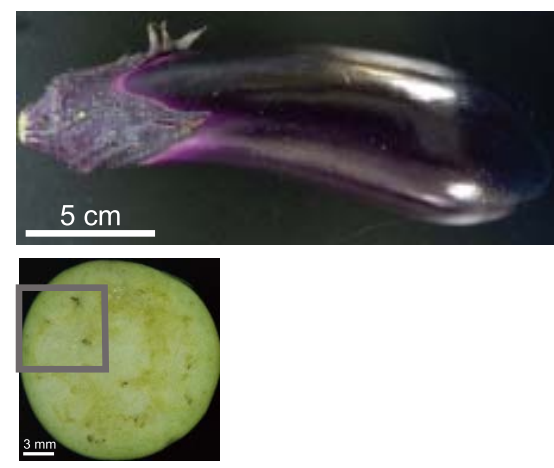

(b)
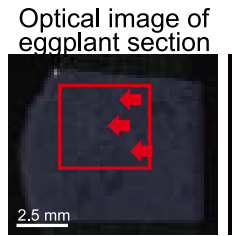

High-power field of red rectangle area

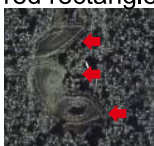

(d) GABA (standard)

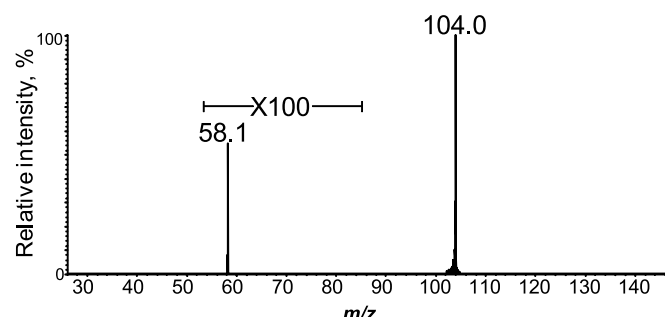

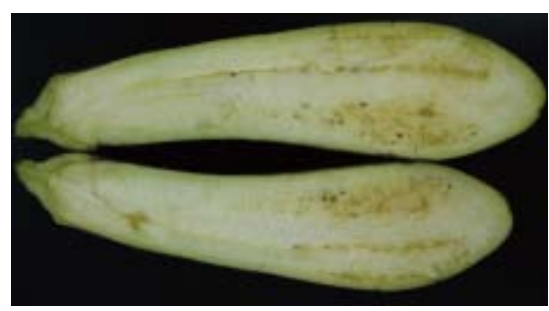

(c)

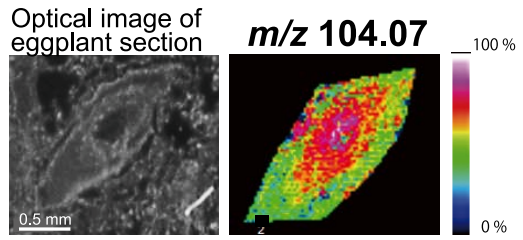

(e) $m / z 104.0$ on tissue

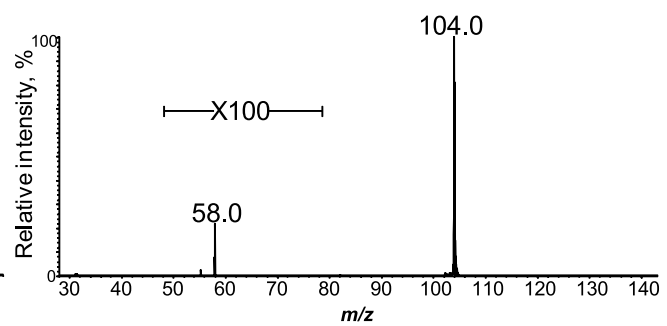

Fig. 1 Optical images of eggplant, the results of IMS and tandem mass analyses. (a) Optical images of eggplant, vertically cut eggplant, and round-cut eggplant. A grey rectangle in a round-cut image shows the region of analyses by IMS. (b) Optical image of eggplant section and ion image of the $\mathrm{m} / \mathrm{z}$ values at 104.07. Red arrows in the optical image show seed locations. Scale bar: $2.5 \mathrm{~mm}$. Reproducibility was confirmed ( $n=3$ ). (c) Optical image of eggplant section and ion image of the $\mathrm{m} / \mathrm{z}$ values at 104.07 with higher spatial resolution at $25 \mu \mathrm{m}$ on a seed. Scale bar: $0.5 \mathrm{~mm}$. (d) The tandem mass spectrum of standard GABA and (e) $\mathrm{m} / z, 104.0$ on eggplant tissue.

benzoic acid (DHB) was purchased from Bruker Daltonics (Bremen, Germany). GABA was purchased from Sigma-Aldrich (St. Louis, MO). All the chemicals used in this study were of the highest purity available.

\section{Sample preparation}

Samples were prepared as previously described, ${ }^{11,26-28}$ with slight modification. Briefly, consecutive $14-\mu \mathrm{m}$ sections of frozen eggplant were prepared using a cryostat (Cryocut CM 1950; Leica Microsystems, Wetzlar, Germany). The sections were mounted on an indium-tin-oxide (ITO)-coated glass slide (Bruker Daltonics). A total of $500 \mu \mathrm{L}$ of DHB matrix solution $(50 \mathrm{mg} / \mathrm{mL} \mathrm{DHB}$ in $70 \%$ methanol $/ 0.1 \% \mathrm{TFA})$ was sprayed uniformly over the sections with a $0.2-\mathrm{mm}$ nozzle caliber airbrush (Procon Boy FWA Platinum; Mr. Hobby, Tokyo, Japan). After the ITO-coated glass slide was dried, it was affixed to the mass spectrometer target plate.

\section{Imaging mass spectrometry}

Imaging mass spectrometry was performed using a MALDI time-of-flight (TOF)/TOF-type instrument (Ultraflex $2 \mathrm{TOF} / \mathrm{TOF}$; Bruker Daltonics). ${ }^{29}$ This instrument was equipped with a 355-nm Nd:YAG laser. The data were acquired in positive ion mode using an external calibration method. In this analysis, signals over the range of $\mathrm{m} / \mathrm{z}, 100-500$ were measured. The MS spectra were calibrated externally using DHB $\left([\mathrm{M}+\mathrm{H}]^{+}, \mathrm{m} / z\right.$ 155.12) and a standard peptide calibration mixture containing $10 \mathrm{pmol} / \mu \mathrm{L}$ of bradykinin peptide fragment (amino acid residues $1-7)\left([\mathrm{M}+\mathrm{H}]^{+}, m / z, 757.40\right)$. The raster scan on the tissue surface was performed automatically by FlexControl and FlexImaging 2.0 software (Bruker Daltonics). The spatial resolutions were set at 200 and $25 \mu \mathrm{m}$. The number of laser irradiations was 200 shots on each spot. Image reconstruction was performed using FlexImaging 2.0 software. 
(a)
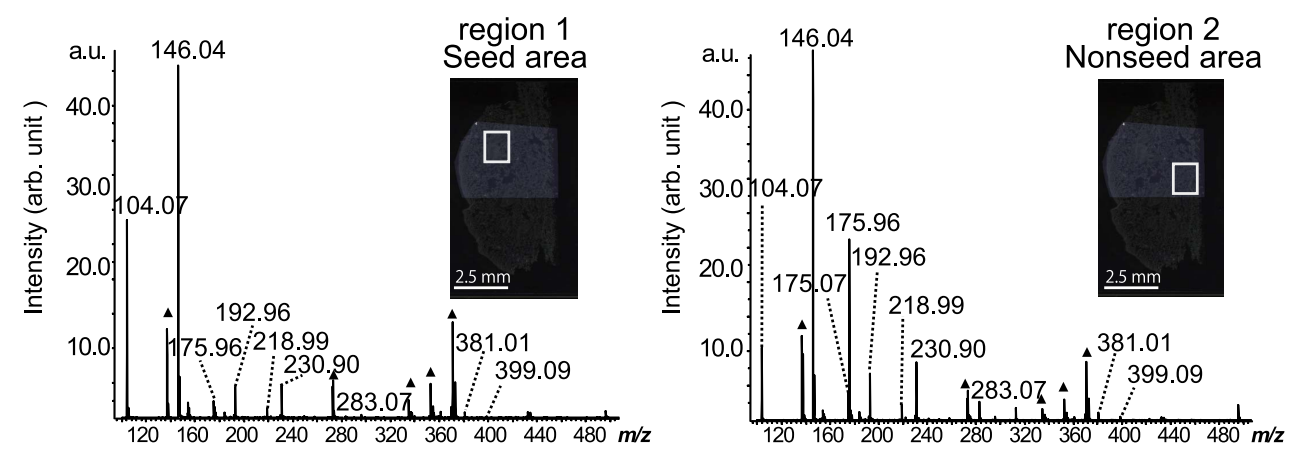

(b)

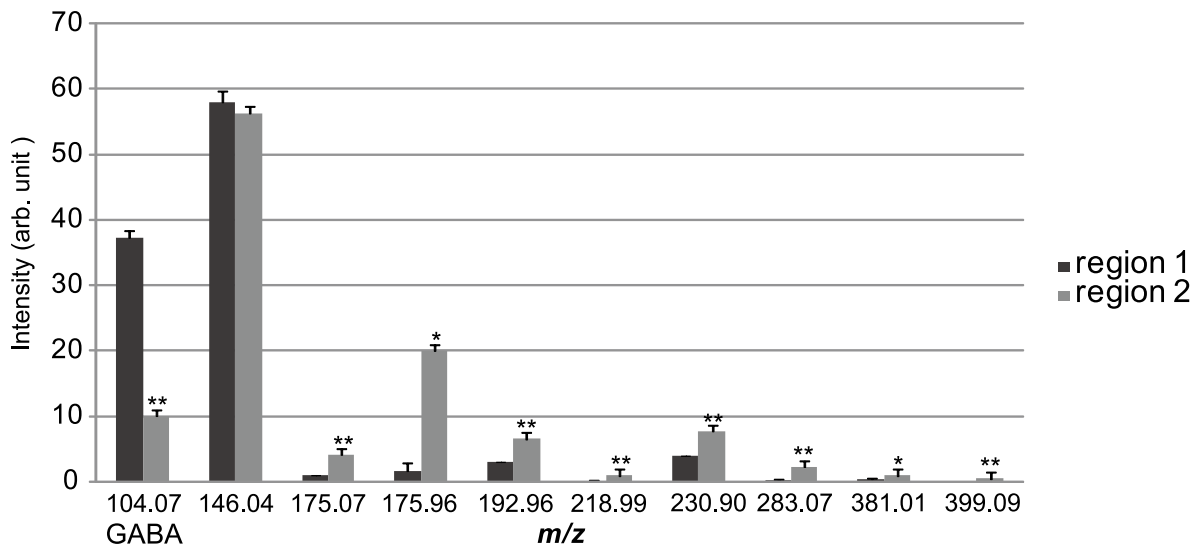

Fig. 2 Results of statistical analyses of two selected regions. (a) The mass spectra of regions of interest 1 and 2 in positive ion mode. The mass spectrum of region 1 shows a seed-specific spectrum, and that of region 2 shows a nonseed area spectrum. The black triangles show the matrix-related peaks. The $\mathrm{Y}$ axis shows arbitrary units calculated by normalizing these spectra. (b) Comparison of relative intensities between regions 1 and 2 . The bar graphs show statistical differences, which were determined using Student's $t$ test $(n=3)$. Differences with a $P$ value of $<0.05$ and $<0.01$ were considered significant. * Indicates a significant difference, $P<0.05$ and $* *$ indicates a significant difference, $P<0.01$.

\section{Tandem mass spectrometry}

Tandem mass spectrometric analyses were performed using an AXIMA-QIT mass spectrometer (Shimadzu, Kyoto, Japan) in positive ion mode. Ionization was performed with a 337-nm pulsed $\mathrm{N}_{2}$ laser. The pulsed gas, argon, was used for collision-induced fragmentation. Precursor and fragment ions obtained by collision-induced dissociation were ejected from the ion trap and analyzed by a reflectron TOF detector. The matrix was $10 \mathrm{mg} / \mathrm{ml} \mathrm{DHB}$ in methanol/0.1\% TFA solution $(1 / 1, v / v)$. The MS spectra were calibrated externally using DHB $\left([\mathrm{M}+\mathrm{H}]^{+}, m / z, 155.12\right)$ and a standard peptide calibration mixture containing $10 \mathrm{pmol} / \mu \mathrm{L}$ of bradykinin peptide fragment (amino acid residues $1-7)\left([\mathrm{M}+\mathrm{H}]^{+}, m / z, 757.40\right)$.

\section{Statistical analysis}

Statistical analysis was performed with StatView 5.0 software (SAS Institute, Cary, NC).

\section{Results and Discussion}

Figure 1(a) shows optical images of eggplant, vertically sliced eggplant, and eggplant in round slices. We transversely sectioned and analyzed tissues near the bottom of the eggplant, as this area contains many seeds. We selected sections that included many seeds and tried to detect GABA and its specific distribution. We measured the sections of eggplant and recorded mass spectra in positive ion mode to detect a GABA-related peak.

Figure 1(b) shows the ion image of $m / z, 104.07$ which has the same $\mathrm{m} / \mathrm{z}$ value as standard GABA. We found that $\mathrm{m} / \mathrm{z} 104.07$ was detected intensely in seeds. To validate the accurate distribution of GABA, we next tried the higher-resolution imaging of a seed on the serial section by setting the spatial resolution at $25 \mu \mathrm{m}$ in positive ion mode (Fig. 1(c)). We confirmed that $\mathrm{m} / z, 104.07$ was distributed in the seed, especially its inner area.

To identify the molecule at $\mathrm{m} / \mathrm{z} 104.07$ that is the specific molecule in seeds, we tried tandem mass spectrometric analyses on the serial tissue section. Because the small molecule has a stable structure, we tried to obtain some fragment ions of the molecule with collision-induced dissociation by AXIMA-QIT. The mass accuracy of AXIMA-QIT is lower than 0.1 Da, thus we showed the $\mathrm{m} / \mathrm{z}$ value with one digit of decimal points. Compared with the spectrum of standard GABA, similar fragment patterns were observed. So, we assigned the peak at $\mathrm{m} / \mathrm{z}, 104.07$ to be GABA as a protonated ion (Figs. 1(d) and 1(e)). By the use of the IMS technique, we found that GABA, one of the functional food factors, has specific localization in seeds.

In addition to GABA, we detected various peaks in eggplant. We selected two regions of interest, regions 1 and 2 , and 
(a)

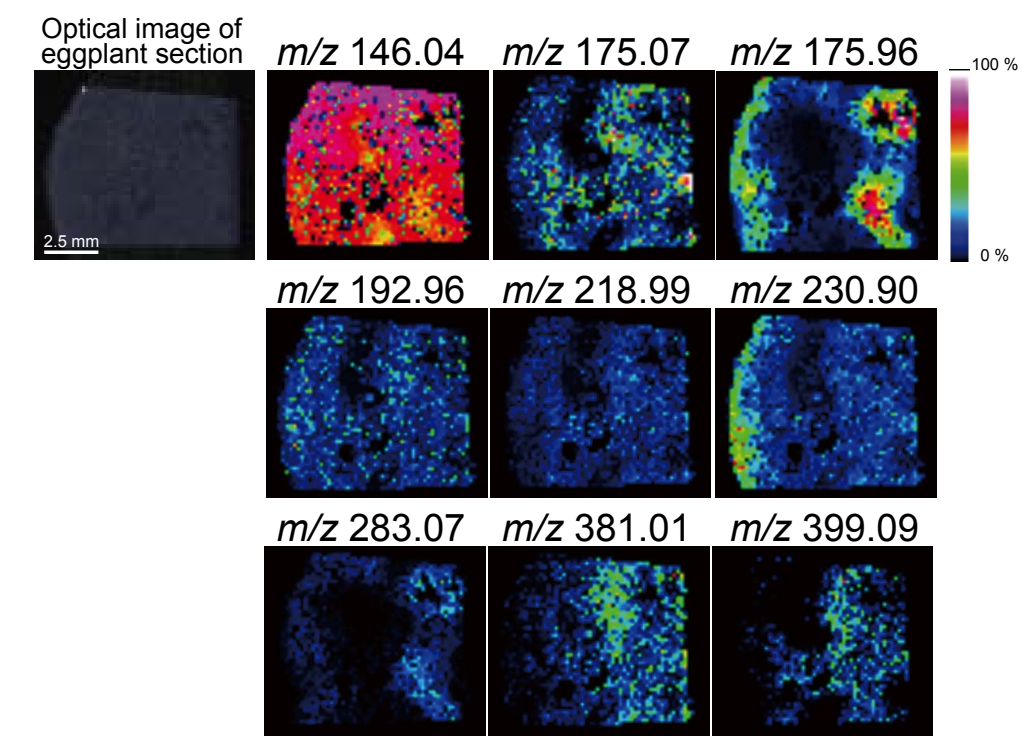

(b)

\begin{tabular}{cccc}
\hline Observed $\boldsymbol{m} / \mathbf{z}$ & Theoretical $\boldsymbol{m} / \mathbf{z}$ & $\Delta$ mass & Possible molecules \\
\hline 104.07 & 104.07 & 0.00 & V-aminobutyric acid + H \\
146.04 & 146.02 & 0.02 & nicotinic acid $+\mathrm{Na}$ \\
175.07 & 175.12 & 0.05 & arginine $+\mathrm{H}$ \\
175.96 & 176.01 & 0.05 & 2-aminobenzoic acid + K \\
218.99 & 219.03 & 0.04 & hexose sugars + K \\
230.90 & 230.99 & 0.09 & citric acid + K \\
283.07 & 283.02 & 0.05 & D-fructose-6-phosphate + N \\
381.01 & 381.08 & 0.07 & sucrose + K \\
399.09 & 399.09 & 0.00 & 2 [hexose sugars] $+\mathrm{K}$ \\
\hline
\end{tabular}

Fig. 3 The distributions of carbohydrates and other amino acids. (a) Ion images of the $\mathrm{m} / \mathrm{z}$ values at 146.04, 175.07, 175.96, 192.96, 218.99, 230.90, 283.07, 381.01, and 399.09 in positive ion mode. (b) The list of annotated peaks.

compared the mass spectrum of each in the positive ion mode (Fig. 2(a)). The mass spectrum of region 1 represented the seed-specific spectrum, and that of region 2 represented the nonseed area spectrum. We clearly observed that these mass spectra differed from each other. As indicated in Fig. 2(a), GABA $(\mathrm{m} / \mathrm{z}$ 104.07) was abundant in seeds, and the intensity of GABA in the region 1 peak was significantly higher than that in region 2. On the other hand, the molecules with $\mathrm{m} / \mathrm{z}$ values of 175.07, 175.96, 192.96, 218.99, 230.90, 283.07, 381.01, and 399.09 were abundant in region 2 with significant differences (Fig. 2(b)). To visualize detected peaks in tissue, we constructed ion images of these peaks (Fig. 3(a)). The molecule at $\mathrm{m} / \mathrm{z}$ 146.04 was distributed ubiquitously. On the other hand, $\mathrm{m} / \mathrm{z}$ 175.07, 192.96, and 218.99 were abundant in the nonseed area. The molecular peak at $\mathrm{m} / \mathrm{z} 230.90$ was localized at the outer area of the eggplant, and the molecular peaks at $\mathrm{m} / \mathrm{z}, 381.01$ and 399.09 were localized in the inner area. The molecular peaks at $\mathrm{m} / \mathrm{z}, 175.96$ and 283.07 were localized in the inner and outer areas.

We assigned these peaks based on the previous reports. ${ }^{24}$ Figure 3(b) lists the possible peaks that were common to IR-MALDI imaging results. The reliability of the peak assignments was expressed by the absolute deviation of observed and theoretical monoisotopic mass values, $\Delta$ mass. Our measurements were done by two-dimensional scanning of tissue sections; $\Delta$ mass $<0.05$ differences might have occurred. Nicotinic acid, which could not be observed in IR-MALDI imaging, was annotated by the comparison with the theoretical mass because eggplant contains large amounts of nicotinoids. As most plant tissues have high concentrations of potassium (as described by the USDA-National Agricultural Lab (http://nal.usda.gov)), potassiated ions were more common than sodiated ones. In our study, we detected four kinds of carbohydrate-related peaks. Amino acids were the second-largest group of primary metabolites observed in previous reports. We did not detect many peaks related to amino acids, though we did observe a few. As shown in Fig. 3(b), we annotated GABA, nicotinic acid, arginine, 2-aminobenzoic acid, hexose sugars, citric acid, D-fructose-6-phosphate, and sucrose as possible entries on the biomolecules list.

As described above, the spatial resolution of the IR laser system has been limited by the focal diameter of the laser beam. Therefore, the spatial resolutions of previous reports were limited to $125 \mu \mathrm{m}$. On the other hand, in our MALDI-IMS, we visualized the localization of metabolites with higher spatial resolution at $25 \mu \mathrm{m}$ (Fig. 1(c)). Therefore, we detected fine-resolution images of GABA and found that GABA was localized inside seeds in eggplant.

Previous reports have shown that eggplant includes GABA, ${ }^{30}$ but its characteristic localization in eggplant has not been reported. In this report, we have shown the specific localization of GABA $(m / z$ 104.07) in eggplant and the possibility that IMS will be a powerful tool for GABA imaging. This result is reasonable because GABA is known to be involved in the regulation of plant development, as well as in defense and other stress responses. ${ }^{31}$ GABA is a nonprotein amino acid component 
present in a wide range of organisms, including bacteria, yeasts, plants, and animals. It is known that GABA intake could also help in the treatment of various neurological disorders such as seizures, Parkinson's disease, stiff-man syndrome, and schizophrenia; ${ }^{32}$ sleeplessness, depression, and autonomic disorders; and chronic alcohol-related symptoms. ${ }^{33}$ It also has an inhibitory action on cancer cells. ${ }^{34}$ Various pharmaceuticals and functional foods have been developed using GABA. Therefore, many plants and agricultural crops have been cultivated for the enrichment of GABA. Our result, showing the characteristic localization of GABA in eggplant, could be applied to create a new functional food with enhanced function. Because it is important for nutritional science to enhance the function of such a factor, distributional information is essential to emphasize the function. In this case, increase or enlargement of seeds can lead to the production of GABA-enriched eggplant.

\section{Conclusions}

In this study, we visualized the distribution of GABA, one of the functional food factors, some other amino acids, and carbohydrates in eggplant sections. We demonstrated that IMS can be applied to the comprehensive analysis of sections obtained from plants for nutritional science and food science. This method will be particularly useful for investigating the distribution of biomolecules such as amino acids and carbohydrates in plants. MALDI-IMS is expected to be used to elucidate the localization of metabolites to explore functional food factors in unused natural resources.

\section{Acknowledgements}

We thank Ms. Mayumi Suzuki for her technical assistance. This work was supported by the Program for the Promotion of Basic and Applied Researches for Innovations in Bio-oriented Industry (BRAIN) to N. Z.; a Grant-in-Aid under the SENTAN program to M. S.; and a Grant-in-Aid for Young Scientists B (21780110) to N. G.-I.

\section{References}

1. J. Lisec, N. Schauer, J. Kopka, L. Willmitzer, and A. R. Fernie, Nat. Protoc., 2006, 1, 387.

2. M. Glinski and W. Weckwerth, Mass. Spectrom. Rev., 2006, $25,173$.

3. T. Soga, Y. Ohashi, Y. Ueno, H. Naraoka, M. Tomita, and T. Nishioka, J. Proteome Res., 2003, 2, 488.

4. I. D. Wilson, R. Plumb, J. Granger, H. Major, R. Williams, and E. M. Lenz, J. Chromatogr., B: Anal. Technol. Biomed. Life Sci., 2005, 817, 67

5. U. Roessner-Tunali, E. Urbanczyk-Wochniak, T. Czechowski, A. Kolbe, L. Willmitzer, and A. R. Fernie, Plant Physiol., 2003, 133, 683.

6. R. Plumb, J. Granger, C. Stumpf, I. D. Wilson, J. A. Evans, and E. M. Lenz, Analyst, 2003, 128, 819 .

7. D. H. Sanchez, M. R. Siahpoosh, U. Roessner, M. Udvardi, and J. Kopka, Physiol. Plant., 2008, 132, 209.

8. S. Sato, T. Soga, T. Nishioka, and M. Tomita, Plant J., 2004, 40, 151.

9. D. S. Cornett, M. L. Reyzer, P. Chaurand, and R. M. Caprioli, Nat. Methods, 2007, 4, 828.

10. S. Shimma, Y. Sugiura, T. Hayasaka, N. Zaima, M. Matsumoto, and M. Setou, Anal. Chem., 2008, 80, 878.

11. N. Zaima, T. Hayasaka, N. Goto-Inoue, and M. Setou, $J$. Oleo Sci., 2009, 58, 415.

12. L. A. McDonnell, S. R. Piersma, A. F. Maarten Altelaar, T. H. Mize, S. L. Luxembourg, P. D. Verhaert, J. van Minnen, and R. M. Heeren, J. Mass Spectrom., 2005, 40, 160.

13. S. N. Jackson, H. Y. J. Wang, and A. S. Woods, Anal. Chem., 2005, 77, 4523.

14. T. Hayasaka, N. Goto-Inoue, N. Zaima, Y. Kimura, and M. Setou, Lipids, 2009, 44, 837.

15. S. W. Cha and E. S. Yeung, Anal. Chem., 2007, 79, 2373.

16. N. Goto-Inoue, T. Hayasaka, N. Zaima, and M. Setou, Glycobiology, 2009, 19, 950.

17. Y. Sugiura, S. Shimma, Y. Konishi, M. K. Yamada, and M. Setou, PLoS One, 2008, 3, e3232.

18. R. M. A. Heeren, Proteomics, 2005, 5, 4316.

19. I. Yao, Y. Sugiura, M. Matsumoto, and M. Setou, Proteomics, 2008, 8, 3692.

20. S. Khatib-Shahidi, M. Andersson, J. L. Herman, T. A. Gillespie, and R. M. Caprioli, Anal. Chem., 2006, 78, 6448.

21. M. L. Reyzer, Y. Hsieh, K. Ng, W. A. Korfmacher, and R. M. Caprioli, J. Mass Spectrom., 2003, 38, 1081.

22. D. Han, H. Y. Kim, H. J. Lee, I. Shim, and D. H. Hahm, J. Microbiol. Biotechnol., 2007, 17, 1661.

23. Y. Li, B. Shrestha, and A. Vertes, Anal. Chem., 2007, 79, 523.

24. Y. Li, B. Shrestha, and A. Vertes, Anal. Chem., 2008, 80, 407.

25. T. Harada, A. Yuba-Kubo, Y. Sugiura, N. Zaima, T. Hayasaka, N. Goto-Inoue, M. Wakui, M. Suematsu, K. Takeshita, K. Ogawa, Y. Yoshida, and M. Setou, Anal. Chem., 2009, 81, 9153.

26. N. Zaima, Y. Matsuyama, and M. Setou, J. Oleo Sci., 2009, $58,267$.

27. S. Taira, Y. Sugiura, S. Moritake, S. Shimma, Y. Ichiyanagi, and M. Setou, Anal. Chem., 2008, 80, 4761.

28. T. Hayasaka, N. Goto-Inoue, Y. Sugiura, N. Zaima, H. Nakanishi, K. Ohishi, S. Nakanishi, T. Naito, R. Taguchi, and M. Setou, Rapid Commun. Mass Spectrom., 2008, 22, 3415.

29. Y. Sugiura, Y. Konishi, N. Zaima, S. Kajihara, H. Nakanishi, R. Taguchi, and M. Setou, J. Lipid Res., 2009, 50, 1776.

30. N. Bouche and H. Fromm, Trends Plant Sci., 2004, 9, 110.

31. H. Ma, Curr. Biol., 2003, 13, R834.

32. C. G. T. Wong, T. Bottiglieri, and O. C. Snead, Ann. Neurol., 2003, 54, S3.

33. S. H. Oh, J. R. Soh, and Y. S. Cha, J. Med. Food, 2003, 6, 115.

34. C. H. Oh and S. H. Oh, J. Med. Food, 2004, 7, 19. 